\section{LE MINIMALISME SATURÉ D'IMAGINATION MORTE IMAGINEZ De SAMUel BeCKetT}

\section{Guillaume Gesvret}

Du "mal vu mal dit" beckettien, on retient souvent le vide et le presque-rien, le silence et le peu-à-voir. Ils se définissent ainsi comme les modalités esthétiques négatives d'une perte et d'un manque partout affirmés: perte des limites dans la dissolution psychotique des premières logorrhées, perte déplorée d'un autre disparu et revenant sans cesse, dans la mélancolie des dernières œuvres. D'un point à l'autre du cheminement complexe de l'œuvre, l'amoindrissement et le ratage deviennent en effet les opérations privilégiées pour témoigner de ce manque. Il s'agit bien de "fermer les yeux pour voir"* Cette nécessité qui parcourt toute l'histoire de l'art moderne, de Maeterlinck au minimalisme contemporain, résonne chez Beckett dans les différents processus d'écriture: comme négativité du regard, elle accompagne tantôt la métamorphose des formes, trop rapide pour fixer l'image de celui qui parle et dont on parle (l'Innommable), tantôt la création d'interstices et de hiatus imprévisibles dans le flux des mots (Comment c'est), tantôt la fragmentation spectrale du corps (Mal vu mal dit).

Chez Beckett, un équilibre instable se crée sans cesse entre apparition et disparition, fixation et mouvement, vie et mort. Cette instabilité permet à l'écriture de continuer avec le même désir de "faire voir" ce qui échappe, d'imaginer là où se défait tout soutien narratif ou illustratif. Ici apparaitt un des principaux paradoxes de l'écriture beckettienne et de la complexité de son économie: si "rien n'est plus réel que le rien", "si ce "rien" non représentable et non symbolisable est identifié au "plus réel" (comme chez Lacan), comment assumer alors la violence positive de ce réel? Comment écrire cette défaillance qui se traduit chez Beckett par la question omniprésente de l'affect et de son expression positive, mélancolique ou angoissée, toujours inattendue et détournée? A la limite ouverte du sens et de la sensation, ces affects sont en effet d'autant plus intenses qu'ils se détournent sans cesse de la règle du moindre et $\mathrm{du}$ pire, ou plutôt y trouvent une place intenable, impossible et pourtant insistante, au point que la lecture fonctionne parfois comme l'attente toujours déjà dupée de ce décalage, de cette effraction de
" (JOYCE, James. Ulysse. Paris: Gallimard, 1929. Repris en "Folio Gallimard", 1996: 58.)

*(Par exemple dans BECKETT, Samuel. Murphy (1938). Paris: Minuit, 1947: 176.) 
l'affect. Si la pénombre, le calme et le silence sont nécessaires, ils impliquent d'autant mieux une violence irrépressible, jamais totalement maîtrisée. Le calme et le silence, dans la torsion subtile d'une prose apaisante, deviennent ainsi le "faux calme du deuil" ou le "silence à l'œil du hurlement" (Mal vu mal dit). Entre le pathos le plus profond et la logique la plus distante, comment l'écriture incarne-t-elle la défaillance de l'affect ressentie en-deçà et par delà les limites subjectives? Dans quel nouveau rapport du corps au langage, du texte à l'image, articuler le défaut et l'excès, l'économie du manque et l'épreuve des corps imaginés/imaginants?

Le titre d'Imagination morte imaginez (1966) résonne comme le "manifeste" de ce rapport paradoxal entre l'épuisement et l'excès. Texte charnière, il se situe entre les premières grandes œuvres théâtrales et narratives, et le minimalisme spectral des vidéos, dramaticules et courts textes de la fin - ou encore sur la zone de passage qui sépare la déconstruction des genres de la problématique des supports. A priori hors de toute narration, il ne s'agit plus ici, prétendument, que de présenter les conditions les plus variées, et peu à peu les plus insoutenables, dans lesquelles deux corps blancs se tiennent dos à dos enfermés dans une rotonde blanche, entourée de murs blancs. Ce dispositif concentre d'ailleurs en sept pages ce que Le Dépeupleur (1970) développera et démultipliera quelques années plus tard. Dans notre texte, et de la même manière, l'imagination ne part de rien ou plutôt d'un presque-rien représenté par un espace inassignable, blanc sur blanc. Dans cet empêchement activement concerté, elle ne se propose rien d'autre au cours du texte que l'image géométrique du lieu et de la posture des corps, qu'elle semble à la fois décrire et inventer: "diamètre 80 centimètres, même distance du sol au sommet de la voûte", "deux diamètres à angle droit $\mathrm{AB} \mathrm{CD}$ partagent en demi-cercles $\mathrm{ACB} \mathrm{BDA}$ le sol blanc. Par terre deux corps blancs." Modèle réduit ou maquette, la mesure de l'espace imaginé déjoue toute vraisemblance: ni à l'échelle d'un lieu réel, chambre ou mausolée, ni même à l'échelle de l'intérieur d'un crâne.

Au fur et à mesure de l'épuisement combinatoire des possibles, la rigueur exhaustive et obsessionnelle du relevé laisse place à l'intermittence d'un éblouissement périodique, nouvelle modalité de l'apparition-disparition:

Attendez plus ou moins longtemps, lumière et chaleur reviennent, sol, mur, voûte et corps blanchissent et chauffent de concert, 20 
secondes environ, tous les gris, atteignent leur palier d'avant, d'où la chute était partie.*

Il arrive aussi, l'expérience le montre, que chute et montée s'interrompent, et cela à n'importe quel palier, et marquent un temps plus ou moins long d'arrêt, avant de reprendre, ou de se convertir, celle-là en montée, celle-ci en chute, pouvant à leur tour soit aboutir, soit s'interrompre avant, pour ensuite reprendre, ou de nouveau se renverser, au bout d'un temps plus ou moins long, et ainsi de suite, avant d'aboutir à l'un ou l'autre extrême.

La "réflexivité" de cette imagination au travail est troublée par un statut changeant, entre la représentation fictive d'une expérimentation sadique sur les corps et le témoignage d'une expérience d'imagination créatrice, d'un "work in progress" dans l'espace mental du crâne. L'obligation mystérieuse de cette invocation s'affirme dans tout le texte à l'impératif, une fois opérés l'évidement de l'espace et le dédoublement premier de l'énonciation: "nulle part trace de vie, dites-vous, pah, la belle affaire, imagination pas morte, si, bon, imagination morte, imaginez." Et plus loin:

Pas d'entrée, entrez, mesurez. [...] Sortez, une rotonde sans ornement, toute blanche, rentrez, frappez, du plein partout, ça sonne comme dans l'imagination sonne."

La situation d'énonciation se déconcerte donc en même temps que la situation des corps. La voix ne correspond plus à une adresse unique: tantôt de soi à soi dans un soliloque où l'impératif évoque les ordres d'un surmoi, tantôt d'un soi anonyme au lecteur ainsi engagé directement dans le travail d'imagination, tantôt d'une pure voix performative à l'œil d'une caméra de surveillance omnisciente et voyeuriste. Cette structure évidée, disjointe et indirecte, conditionne la nouvelle imagination invoquée par le titre. Plainte de l'impuissance à voir, et de la déchéance d'une vision intérieure, ou tentative critique de trouver un autre rapport à l'image hors d'une pure intériorité subjective, il s'agit toujours de construire une nouvelle reconnaissance là où les repères se suspendent, "faire l'image" dans la négativité créatrice d'une nouvelle écriture du corps. Aussi, l'évidement et la disjonction se mettent en scène, et l'écriture devient dispositif. Non pas l'oubli de la représentation dans une pure littéralité, mais l'engagement des éléments qui constituent la représentation dans une présentation problématique et réflexive. En effet, tout commence souvent chez Beckett par la disposition des corps et du lieu, sans visée immédiatement et exclusivement nar- 
rative ou dramaturgique. On peut donc entendre ce "dispositif" dans la spécificité de sa connotation scientifique, autant qu'esthétique (les "installations" contemporaines tout juste évoquées), ou encore politique (comme mise en scène ironisée d'un pouvoir et de ses contraintes). Le travail d'écriture se présente ainsi dans le jeu des dissociations, entre décomposition et recomposition des conditions minimales de l'écriture: une voix, un œil, deux corps, un lieu sans nom et un éblouissement répété. Sans genre défini, le dispositif affirme sans cesse son rapport au dehors d'une pure opération littéraire, poème ou fable. Comme on le verra, le texte fonctionne aussi bien comme protocole expérimental, relevé physico-chimique de cette expérience, instruction pour un schéma à venir (inimaginable à la seule lecture), projet d'un film, d'une mise en scène ou d'une installation plastique.

Dans ce dispositif, l'épuisement des référents stables de l'écriture et de la visibilité de l'espace se joue comme toujours chez Beckett avec l'épuisement des corps: "fermer les yeux pour voir" tout en éprouvant les corps. "Eprouver" s'entendra alors au double sens, actif et passif, de mettre à l'épreuve et de ressentir. L'épreuve s'élabore en effet chez Beckett à partir de cette ambivalence: à la fois construite et ressentie, entre contrainte logique et sensation physiologique. Questionnement physique ("un corps peut-il soutenir de telles conditions dans un tel espace") inséparable d'une critique métaphysique: chez Beckett, le "corps" comme identité charnelle du sujet n'est jamais centré et plein, mais toujours déjà dédoublé ou décentré. Ambiguïté de cette "question" du corps, entre torture et travail critique.

Il s'agit donc de voir comment joue l'affect dans la mécanique bien close et a priori non subjective de la logique géométrique ou combinatoire. A cet égard, Imagination morte imaginez possède une structure très singulière: non seulement le lieu des corps et le rapport voix-image sont pris dans une écriture de l'inassignable, mais les corps eux-mêmes sont au début du texte à peine évoqués: posés comme de simples hypothèses, ils sont aussitôt oubliés. C'est ainsi dans cet oubli et dans la disparition de ces deux corps, articulés au suspense de leur réapparition (ultime résidu de narration), que la logique des combinaisons se laisse déborder imperceptiblement par la violence d'affects anonymes. Sur fond d'inassignable et de disparition, et dans la logique mathématique la moins affectée, l'espace de l'expérimentation laisse apparaître des signe de vie, sensibles ou plus métaphoriques: 
Par de tels hauts et bas, remontées et rechutes, se succédant dans des rythmes sans nombre, il n'est pas rare que le passage se fasse, $\mathrm{du}$ blanc au noir et de la chaleur au froid et inversement. Seuls les extrêmes sont stables, comme le souligne la pulsation qui se manifeste lors des pauses aux paliers intermédiaires, quelles qu'en soient la durée et la hauteur. Frémissement alors. [...]

Et le plus souvent, quand la lumière se met à baisser, et avec elle la chaleur, le mouvement se poursuit sans heurt jusqu'au noir fermé et au degré zéro. [...]

Suit dans l'ordre des fréquences la chute ou montée avec temps d'arrêt plus ou moins longs dans ces gris fiévreux sans qu'à aucun moment le mouvement soit renversé. [...]

N'empêche qu'une fois l'équilibre rompu, celui du haut comme celui du bas, le passage au suivant est variable à l'infini.'

L'espace de la mesure est ainsi comme "enveloppé par la possibilité d'une rupture"1 (Blanchot), toujours déjà pris dans un devenir-affect mystérieux. Ces évocations se jouent à la limite d'une métaphore de la condition humaine ("chute", "équilibre rompu" cause d'une "variation infinie") et de la pure sensibilité d'un corps anonyme (rythme cardiaque d'une "pulsation", "frémissement" et "fièvre"). L'affect semble se créer sans cause directe ni référence à un corps délimité. Il fait alors effraction à la manière d'un symptôme dans le calcul sans faille des combinaisons. Le symptôme se caractérise en effet chez Freud par son travail, semblable à celui du rêve: né de l'oubli, de la "nuit" de la conscience et de la défaillance d'un savoir unifié, il s'insinue dans la forme même du corps et du langage. Compromis déjouant la censure du désir inconscient, il apparaît comme questionnement non résolu, présentation inquiète qui déconcerte la représentation et le sens de son apparition ou de sa possible interprétation. Le symptôme permet cependant de soutenir la "maladie" et de ne pas s'effondrer, ne défait le refoulement que partiellement, en même temps qu'il interdit toute "synthèse symbolique" et toute "interprétation totalisante". En écho avec le

\footnotetext{
${ }^{1}$ Sur le travail comblant de la logique chez Lautréamont, et ce "peu de repos ménagé à l'œil placé en face d'un bloc compact": "Limpression que doit ressentir tout premier lecteur est nécessairement celle d'un texte où la plus grande clarté, la logique la plus rigoureuse coïncideraient avec une confusion complète et l'impossibilité de 's'y retrouver' [...]. Il arrive que la lenteur, la patience, la solidité du langage logique soient elles-mêmes comme enveloppées par la possibilité d'une rupture: ces qualités raisonnables menacent sans cesse la raison, car elles recèlent, au fond de leur sérieux, le sarcasme qui détruit ce sérieux et, au fond de leur tranquillité, l'irruption bouleversante qui rend la tranquillité impossible". BLANCHOT, Maurice. La part du feu. Paris: Gallimard, 1949: 161/162 (je souligne).
}

(lbidem: 54.)

" (FÉDIDA, Pierre, "La sollicitation à interpréter". In: $L^{\prime}$ Ecrit du temps, ${ }^{\circ} 4$. Paris: Minuit, 1983: 13.) 
-(DIDI-HUBERMAN, Georges. Devant l'image. Paris: Minuit, 1990: 192-195.)

(ARTAUD, Antonin. Le théâtre et son double (1938). In: Oeuvres complètes. IV. Paris: Gallimard, 1964: 154.)

(BECKETT, Samuel. Imagination morte imaginez. op. cit.: 55.$)$ travail de l'image picturale, Georges Didi-Huberman a tiré parti de cette définition du symptôme dans ses analyses esthétiques:

Le symptôme ne se donne à voir que dans la déchirure et la défiguration partielles qu'il fait subir au milieu dans lequel il advient. [...] Symptôme nous dit l'infernale scansion, le mouvement anadyomène [...] de la présence dans la représentation. Il nous dit l'insistance du singulier dans le régulier, il nous dit le tissu qui se déchire, la rupture d'équilibre et l'équilibre nouveau, l'équilibre inouï qui bientôt de nouveau va se rompre.

Dans notre texte, les moments symptomatiques interrompent ainsi l'invention de l'image et l'épuisement des possibles, comme si le travail de l'imagination était aussi concerné par la négativité du symptôme et la matérialité précaire qu’il donne à voir, à sentir. Les deux corps, un temps oubliés, se font entendre dans ces détails du texte, modalité textuelle et logique d'un tressage où l'affect apparaît par intermittence, là où s'ouvre un passage dans la trame. Les corps s'absentent mais ne cessent d'être implicitement épuisés, éprouvés par la succession des changements de conditions, entre hypothèse et réalisation. Cette épreuve latente creuse ainsi l'imminence de la ré-apparition des corps et semble laisser échapper ces "spectres plastiques", ' comme autant d'intensités évanouissantes, témoignages précaires d'un affect. Dans cette structuration symptomatique, l'apparition de telles forces relève toujours d'une surprise et d'un écart, comme en-deçà de la constitution des corps, ou bien au delà, n’appartenant plus aux corps, les traversant dans cet élan "trop grand pour soi” (Deleuze sur l'affect). L'épuisement s'affirme en somme dans une saturation double et paradoxale: l'énumération obsessionnelle qui cherche à combler exhaustivement chaque faille, empêchant le travail de l'imagination (premier sens d'une saturation négative) n'aboutit pas à l'anéantissement des corps et de l'espace. Au contraire: dans la négation généralisée et la "mise en latence" des corps, dans le compte statistique des différentes conditions (décompte implicite d'une fin imminente), un mourir vivant et contradictoire sur-vient, vitalité excessive et "convulsive" soutenue par l'harmonie rythmique de la prose:

Mais quels qu'en soient les hasards, le retour tôt ou tard au calme temporaire semble assuré, pour le moment, dans le noir ou la grande blancheur, avec température afférente, monde à l'épreuve encore de la convulsion sans trêve. Retrouvé par miracle après quelle absence dans des déserts parfaits il n'est déjà plus tout à fait le même [...]. 
Une chose sans nom sature donc positivement, entre angoisse et harmonie. Elle excède le plan neutre du "désert parfait" et le cadre d'une scientificité juridique ("température afférente"), en leur insufflant un devenir-corps. Cette apparition trouble d'autant plus le "calme" du dispositif qu'elle donne l'occasion d'un nouage singulier entre la douceur poétique et rythmique de la matérialité sonore et la violence de la représentation, ici quasi-abstraite et sans sujet défini, d'un "corps-monde" convulsif. Cette "convulsion" (c'est-à-dire à la fois la paralysie et l'agitation, l'immobilité et le spasme saccadé, la contorsion douloureuse) s'exprime en effet dans le ralentissement d'un rythme ponctué d'échos sonores particulièrement travaillés: entre la fluidité des "liquides" [l] et $[\mathrm{r}]$, et le tranchant plus cruel des [v], d'une phrase à l'autre: "épreuve, convulsion, trêve, retrouvé". L'évocation de cette violence physique, dans un "lyrisme hors du soi", " fait contrepoint avec l'éblouissement qui revient et se découvre peu à peu comme l'objet insaisissable du texte. L'un et l'autre jouent comme autant d'engagements inouïs du corps dans la plasticité de l'écriture et la mise en crise de la représentation. A quoi attribuer cette "convulsion" qui fait signe au centre du texte, et s'y présente comme le nom mystérieux d'un excès entrevu? Est-ce la description d'un "monde" intérieur, d'une crise d'épilepsie face à l'éblouissement répété? Ou bien la convulsion du monde comme extériorité historique, à la fois éprouvant et mis “à l'épreuve"? Ou s'agit-il enfin de la recherche à la surface de l'écriture, dans ses plis et trouées, d'une respiration lumineuse, d'un éblouissement convulsif comme le témoignage même d'un dehors plus absolu - au monde et à tout sujet?

La "convulsion sans trêve" apparait donc pour faire symptôme dans un arrêt de la lecture, arrêt paradoxal devant cette force "sans trêve". Entre dedans et dehors, entre présentation et représentation, la force de l'affect traverse ainsi tout le texte en troublant ses repères, en modifiant son rythme, sa forme et ses interprétations possibles. D'un hiatus imprévisible à l'autre, le texte est comme "saturé" de ces affects symptomatiques dans toute l'ambiguïté paradoxale du terme: ainsi, la "saturation" est par exemple au sens chimique l'action de dissoudre dans un liquide la masse maximale d'une substance, à une température et sous une pression données, ou encore l'état d'équilibre ainsi obtenu. "Un corps est saturé d'un autre quand il est combiné avec tout la quantité possible de celuici" (Littré). A cette saturation comme équilibre atteint dans l'épui-
(MARTIN, Jean-Pierre. " vite, vite avant de pleurer': L'absolu de l'affect dans Textes pour rien". In: Samuel Beckett Today/Aujourd'hui. $\mathrm{n}^{\circ}$ 10. Amsterdam/New York: Rodopi, 2000: 103.) 
sement des possibles, s'ajoute celle qu'on attribue à la couleur ou au son: saturation colorée ou électro-acoustique, quand le signal émis dépasse la limite et crée une distorsion, une intensité excessive qui trouble ou choque la perception. Est donc saturé ce qui atteint un seuil menacé par un débordement (point de saturation) où la matière cesse de se dissoudre et apparaît comme un symptôme, où la sensation devient trop grande. A la fois l'équilibre, la tension maximale donc, mais aussi, et comme implicitement, ce qui toujours déjà dépasse la limite, l'excès, la surabondance. Même dans le sens le plus commun, "être saturé" contient cette équivoque première, la mise en devenir de ces deux sens: tension extrême et perte de maîtrise, équilibre et déséquilibre, comblement fini et possibilité menaçante d'un débordement infini. Dans notre texte, l'épuisement est bien cette accumulation, ce travail d'une limite où l'excès (de matière non dissolue, de sensation non épuisée) risque d'apparaître et d'échapper, dans une intensité d'autant plus évanouissante et donc d'autant plus grande, que les conditions de son empêchement sont fortes. Ainsi, les symptômes se présentent moins comme une image figurative à décrire, qu'à la manière d'un excès où le texte et l'image tendent à se confondre dans une métamorphose de l'écriture. "Infra-langage qui s'élabore à la limite de la chair et du monde"2 (Barthes), la métamorphose est ici l'effraction symptomatique d'une matérialité instable qui fait défaillir le sens et fait s'arrêter le regard.

Cette métamorphose reste cependant nécessairement inachevée, et les corps font retour dans la représentation (dans l'imagination, l'expérimentation) aux deux dernières pages:

Toujours par terre, plié en trois, la tête contre le mur à $\mathrm{B}$, le cul contre le mur à $\mathrm{A}$, les genoux contre le mur entre $\mathrm{B}$ et $\mathrm{C}$, les pieds contre le mur entre $\mathrm{C}$ et $\mathrm{A}$, c'est-à-dire inscrit dans le demi-cercle $\mathrm{ACB}$, se confondant avec le sol n'était la longue chevelure d'une blancheur incertaine, un corps blanc finalement de femme. [...] Blanc aussi à l'égal du sol le partenaire. Sur le flanc droit donc tous les deux têtebêche dos à dos. Présentez une glace aux lèvres, elle s'embue. De la

\footnotetext{
${ }^{2}$ Sur la métamorphose et le style, cf. BARTHES, Roland. Le Degré zéro de l'écriture. Paris: Seuil, 1953: 12-13: "Le style est le terme d'une métamorphose aveugle et obstinée, partie d'un infra-langage qui s'élabore à la limite de la chair et du monde". Il "compose son opacité à partir d'une certaine expérience de la matière", il est une "équation entre l'intention littéraire et la structure charnelle de l'auteur", "un phénomène de densité, car ce qui se tient droit et profond sous le style, rassemblé durement ou tendrement dans ses figures, ce sont les fragments d'une réalité absolument étrangère au langage".
} 
main gauche chacun se tient la jambe gauche un peu au-dessous du genou, de la droite le bras gauche un peu au-dessus du coude.*

L'écriture maintient donc la représentation d'une "scène" avec (BECKETT, Samuel. Imagination morte imaginez. op. cit.: 56.$)$ la précision ultra géométrique d'une double posture, reliant et séparant à la fois une femme et son "partenaire" - version érotique de "l'empêchement" et ironie sadique du dispositif. Contorsion oscillant entre jeu et torture, elle est une nouvelle création dans l'éventail des postures beckettiennes. Dans ce reste de représentation, le rapport des deux corps est a priori empêché à l'extrême, et même condamné, comme dans le chant XX de l'Enfer de Dante où l'on trouve la même posture dos-à-dos pour décrire les damnés. Pourtant, ce rapport semble s'affirmer malgré tout dans la négation même de tout rapport: ainsi, les deux corps réussissent encore à se tenir l'un à l'autre dans cette étonnante posture. De même, au sujet de l'écarquillement des yeux: "Jamais les deux regards ensemble sauf une seule fois une dizaine de secondes, le début de l'un empiétant sur la fin de l'autre". Une activité commune, à peine perceptible, et sans utilité se découvre derrière l'apparente inactivité neutre et morbide, inertie engagée dans l'écho convulsif de l'écriture du symptôme. Ce reste en exception fonctionnerait alors comme la modalité logique de l'excès. Impossible, illogique, l'expérience d'une rencontre apparaît de manière encore plus mystérieuse à la fin du texte. Les dernières lignes retentissent de cette actualisation intenable du symptôme: le point final coïncide avec le suspens intensif d'un acte en commun, dans l'imminence, le retard, le surgissement in extremis de l'évènement:

Laissez-les là, en sueur et glacés, il y a mieux ailleurs. Mais non, la vie s'achève et non, il n'y a rien ailleurs, et plus question de retrouver ce point blanc perdu dans la blancheur, voir s'ils sont restés tranquilles au fort de cet orage, ou d'un orage pire, ou dans le noir fermé pour de bon, ou la grande blancheur immuable, et sinon ce quils font.

* (Ibidem: 57.)

On remarque comment les clivages et ajouts successifs dans la phrase, plus ou moins adéquats syntaxiquement (hyperbates), empêchent de raccorder explicitement les deux expressions "plus question de voir" et "ce qu'ils font", retenant la conclusion entre sa négation logique et l'affirmation latente, suspendue, de son contenu: l'acte possible, la rencontre. L'opposition entre le virtuel et l'actuel devient indécidable, le virtuel "s'incarnant" dans cette affirmation symptomatique et mystérieuse. Une vérité ironique et 
tragique de "l'impossible rapport sexuel" prend corps à la surface de l'écriture dans un acte paradoxal ("ce qu'ils font"), et fait signe rétrospectif pour une nouvelle lecture de l'œuvre: celle qui découvre non pas un contenu signifiant, mais le rapport dans le non rapport, la continuité nouvelle assumant la discontinuité, la représentation sauvée et exceptée de corps vivants malgré tout: "présentez une glace aux lèvres, elle s'embue". *

Avant même cette fin ambiguë, il faut noter que l'enfermement des corps (dans un crâne-mausolée ou dans le lieu sans lieu d'une étreinte impossible) est conditionné par une autre logique géométrique elle-même ambiguë. La géométrisation est ainsi exploitée à un tel point que l'imagination du lecteur peine à se représenter l'articulation des deux corps. Nouveau travail de mise en crise, d'ouverture des limites textuelles traditionnelles à la possibilité d'un devenir plastique, ici par exemple celle d'un schéma. La négativité créatrice de l'écriture comme travail du symptôme touche alors le statut même du texte. Ces descriptions ou instructions géométriques, qui ne peuvent faire image toutes seules sans leur nécessaire mise en forme schématique, ouvrent le texte sur un possible non réalisé, excessif, évoqué puis oublié. La mise en mesure géométrique de l'espace des corps joue ainsi sur la multiplicité de statuts latents et en devenir, hors de la clôture du texte. On pourrait d'ailleurs noter que cette modalité violente et complexe de la représentation des corps est le plus souvent "textuelle" chez Beckett, et qu'elle ne se retrouve pas ou peu dans les œuvres qui proposent des images visuelles: les pièces ou vidéos ne sont en effet jamais de simples illustrations des textes. L'expressivité visuelle, quand elle apparaît dans les vidéos, s'engage ainsi tantôt dans un isolement fragmentaire (la bouche de Pas moi qui épuise toutes les grimaces possibles dans sa logorrhée), tantôt dans une répétition (celle du déhanchement des danseurs de Quad). Limage corporelle proposée par Imagination morte imaginez apparaitrait plutôt chez certains vidéastes des années 1970: les variations sur la posture du corps, souvent celui de l'artiste lui-même, y deviennent une véritable écriture, exploration visuelle, banale ou monstrueuse, de ses potentialités plastiques dans la destitution de toute narration (on pense aux références les plus connues: Bruce Nauman, Gary Hill ou Dan $\mathrm{Graham}^{3}$ ). Moins le modèle ou l'influence directe mo-

\footnotetext{
${ }^{3}$ La situation optique d'Imagination morte imaginez évoque par exemple cette vidéo/performance de Dan Graham (Body press, 1972), où les corps d'un hom-
} 
tivant la création de ces œuvres, Imagination morte imaginez crée avec elles l'écho et la résonance où persiste la singularité des démarches, d'un espace d'écriture à l'autre. Le travail contradictoire, qui consiste ici à dire la violence de l'affect dans l'indifférence d'une logique, nécessite sans doute une lecture silencieuse du texte, sensible à l'évolution et à l'ouverture d'une logique.

Aussi, il ne s'agit pas de distinguer simplement la présentation plastique du symptôme et sa représentation logique, le moment de "convulsion" dans l'harmonie poétique et la description géométrique d'un rapport intenable. En effet, la représentation alterne sans cesse avec ces moments plastiques où une violence sans sujet fait surface, se présentant au détour d'une phrase. Même après le "retour" du couple des damnés enfermés dans la rotonde, on retrouve partout ces signes paradoxaux. La tension est dès lors permanente entre la littéralité sensible du texte et l'image des corps "inertes comme en pleine convulsion". En témoigne la caractérisation de plus en plus incarnée de l'environnement lumineux: "Dans cette lumière agitée, au grand calme blanc devenu si rare et bref”, * "entre leur immobilité absolue et la lumière déchaînée le contraste est frappant", "voir s'ils sont restés tranquilles au fort de cet orage, ou d'un orage pire".* La continuité du texte est parfois littéralement trouée par un débordement physique ou physiologique, par exemple un trop-plein de chaleur: "Des extrêmes tant qu'ils persistent, la stabilité est parfaite, ce qui colonne chaleur peut paraître étrange, dans les premiers temps". ${ }^{4}$ D'une force croissante et continue, ces signes sont pris dans une temporalité complexe: elle relève à la fois de l'approche progressive d'un état de violence extrême dans une imminence de plus en plus en plus incarnée, mais aussi bien

me et d'une femme sont enfermés nus et debout dans un cylindre, entourés d'un grand miroir courbe recouvrant l'intérieur, chacun muni d'une caméra et pivotant sur place par intervalle régulier. Les images obtenues apparaissent simultanément sur deux écrans-vidéos (un écran de "contrôle" pour chaque caméra): tantôt l'image propre du filmeur au travail dans le miroir, tantôt celle de l'autre corps, tantôt le reflet de l'un ou l'autre déformé par la courbure du miroir. Dans cette exploration visuelle de l'espace des corps, différence sexuelle et différence des statuts de l'image varient ainsi en circuit fermé et continu, au risque de l'indiscernable. On pourrait aussi penser à l'image frappante du corps recroquevillé de Gary Hill dans Incidence of catastrophe (1988) d'après Thomas l'obscur de Blanchot, très proche des figures du corps-déchet beckettien.

${ }^{4}$ Ibidem: 53. Je souligne cette interruption; elle évoque autant une "colonne d'air" que la "colonne" d'un classement des différents niveaux de température, entre intensité sensible et relevé objectif.
(ARTAUD, Antonin. Van Gogh le suicidé de la société (1947). Paris: Gallimard, 2001: 41.)

* (BECKETT, Samuel. Imagination morte imaginez. op. cit.: 56.$)$

(Ibidem: 57.)

(Ibidem: 57.) 
(Ibidem: 57.)

(lbidem: 56.$)$

- (FREUD, Sigmund. Inhibition, symptôme, angoisse (1926). Paris: PUF, 2005.)

- (LACAN, Jacques. Séminaire $X$ - L'angoisse (1962-63). Paris: Seuil, 2004.) de traces "déjà-là" qui interrompent le fil linéaire de la lecture devant la densité inquiète et distante du symptôme.

Dans le mystère de cet éblouissement sans source, le débordement semble approcher tout en produisant toujours déjà ses effets. Il passe ainsi à la fois par les corps et par le cadre troué de l'espace d'écriture, tous emportés dans le même devenir. Objet sans nom, il ne semble pouvoir se dire qu'entre le déchaînement et la faiblesse, dans l'accumulation impossible de sensations contradictoires: "Laissez-les là, en sueur et glacés, il y a mieux ailleurs." L'épreuve de l'invivable et de la saturation positive que les corps subissent par-delà leurs "possibilités" est paradoxalement encore le signe d'une vie possible:

Ils passeraient bien pour inanimés sans les yeux gauches qui à des intervalles incalculables brusquement s'écarquillent et s'exposent béants bien au-delà des possibilités humaines. Bleu pâle aigu l'effet en est saisissant."

"L'œil béant" revient jusque dans les derniers textes de Beckett comme la métonymie saisissante de cet objet auquel il s'expose. Métonymie suspendue, puisque l'œil est séparé de cet objet qui lui donnerait sens, elle devient d'autant plus angoissante. C'est 'l'œil du hurlement" déjà cité, représentation poétique et nécessairement indirect de l'objet angoissant. Chez Freud, l'angoisse est l'affect éprouvé face à l'excès de la demande pulsionnelle et de la jouissance entrevue, face à un trop-plein (der Uberschuss) de libido inutilisée. ${ }^{*}$ Lacan la définit en particulier comme un "signal du réel”, signal de cette altérité irréductible, irreprésentable et monstrueuse du réel. Menace d'un débordement, d'une "négation de la castration", l'angoisse advient alors quand "le manque vient à manquer". " Lacan ajoute d'ailleurs à l'analyse freudienne d'une angoisse phénoménologique et ressentie, la dimension logique d'une angoisse comme temps non repérable mais nécessaire à la constitution du désir. ${ }^{5}$ Il s'agirait comme chez Beckett de tenir un double mouvement: à la fois donner lieu à cet excès insistant en deçà du désir et maintenir une distance, creuser un manque. Sortir de l'angoisse pour désirer à nouveau nécessite alors de créer le "manqueà-être" sur fond de cet excès, s'affrontant à ce qui au sein de l'angoisse relève du "plus réel". Un manque actif donc qui constitue le désir et se joue à sa cause même. On s'éloigne dès lors de ce que

5 “Le temps de l'angoisse n'est pas absent de la constitution du désir, même si ce temps est élidé, non repérable dans le concret” (Ibidem: 204.) 
Deleuze dénonçait comme désir "soumis" au manque, et plus proche de "l'évidement actif" que le philosophe définissait lui-même comme le moteur de l'écriture - on pourrait noter ici la proximité de Deleuze et du dernier Lacan, leur questionnement commun sur le désir comme "désir de rien", construction active à l'épreuve d'un manque constitutif, d'une violence première, définissant le sujet comme effet et résidu. ${ }^{6}$

Imagination morte imaginez met en scène ces effets incertains et décentrés d'un en deçà du sujet, là où l'imagination devient questionnement sans support fixe d'un corps inconnu et en devenir. Dans la discontinuité mouvante de l'écriture, les symptômes évoqués sont produits par l'écriture comme des hiatus incarnés, signes précaires d'une angoisse approchée et distanciée: approchée, parce qu'elle donne accès au plus réel dont l'écriture cherche à créer le signe et à faire l'image; distancié, justement parce que le langage, l'imagination, la représentation, s'y perdent définitivement. Entre l'attention inquiète à cette violence qui insiste "sans trêve" et l'accalmie nécessaire pour témoigner du "déchaînement" vital ou catastrophique, les symptômes d'écriture deviennent des "points de capiton": cette métaphore lacanienne décrit bien le symptôme comme trouée dans la continuité rassurante d'une logique et soutien paradoxal qui permet de continuer. Ces points en mouvement, sans lieu fixe, sont chez Beckett une ponctuation matérielle, interstices à peine localisables dans le mouvement des devenirs, dans l'intermittence qui maintient la tension du désir à la limite de l'effondrement.

Si le problème est de donner lieu à l'excès du réel entrevu dans l'affect, le "minimalisme" beckettien s'explique sans doute mieux dans ce rapport précaire à une double saturation. Il s'agit d'abord de soustraire, d'évider l'excès négatif des images aliénantes, des fantasmes qui recouvrent le réel, des discours et des représentations qui nous saturent, pour trouver ce lieu non reconnaissable de l'expérimentation beckettienne, blanc sur blanc ou noir du crâne. Enjeu esthétique et critique, cet évidement ouvre un espace minimal et paradoxal pour soutenir l'épreuve du réel. L'écriture creuse et libère ainsi cet espace d'une autre saturation, positive et risquée, confrontation au réel qui échappe. Le lieu du "moindre" et du "presque rien" est donc moins le résultat d'un épuisement achevé que l'espace plus intense d'un entre-deux et d'une tension maximale.

\footnotetext{
${ }^{6}$ Le sujet "est sans identité fixe, toujours décentré, conclu des états par lesquels il passe”. DELEUZE, Gilles. L'Anti-CEdipe. Paris: Minuit, 1972: 27.
} 
- (BECKETT, Samuel. "Peintres de l'empêchement" (1948). In: Le monde et le pantalon suivi de Peintres de l'empêchement. Paris: Minuit, 1991.)

(DELEUZE, Gilles. L'Epuisé, introduction à Quad. Paris: Minuit, 1992.)

- (Lettre de Beckett à Avigdor Arikha. 16 mars 1965. Citée par KNOWLSON, James. Beckett. Paris: Acte sud, 1999: 673.)
La saturation beckettienne opère dans l'ambivalence propre à d'autres notions souvent invoquées pour analyser son œuvre: "l'empêchement" analysé par l'auteur chez les frères van Velde* (comme impuissance et dessaisissement créateur) ou "l'épuisement" deleuzien* (comme fatigue et déploiement des possibles). Imagination morte imaginez est donc un exemple particulièrement dense de cette économie singulière, où la négativité crée un nouvel espace d'imagination et d'énonciation où faire jouer la force de l'affect: “je veux mettre de la poésie dans le théâtre, une poésie en suspens dans le vide et qui prenne un nouveau départ dans un nouvel espace" (Beckett). Articulation singulière d'un manque créateur à l'excès de l'affect, l'économie esthétique de Beckett s'élabore dans un minimalisme saturé, dans une "saturation par transparence". ${ }^{8}$ L'œuvre crée alors ses propres conditions pour mieux faire jouer l'ouverture d'un hiatus et d'une liberté au sein même de sa clôture: "J'ai bouclé la rotonde. Pour en être délivré. Mille mots, Six mois de ratures".

Une fois défait le principe d'une représentation unifiée (figurative, narrative ou dramaturgique), l'écriture peut donc mettre en devenir à la fois sa logique formelle et l'expression de l'affect. D'un évènement d'écriture (plastique et logique) à l'autre, elle engage dans un même mouvement à la fois la distance du dispositif, qui évide et disjoint les conditions d'écriture, et le travail de la surface, du rythme, de l'incarnation. Oscillation sans cesse risquée entre le partage de l'espace au risque du sens et la présence d'une matérialité intensive, excessive et symptomatique. Le titre "Imagination morte imaginez", formulé d'une traite, sans virgule, fait alors signe pour cette dialectique sans étapes ni résolution, recherche jamais achevée de nouveaux rapports dans le flux des mots et des images.

Par sa radicalité, l'exploration beckettienne nous oblige à comprendre comment certains enjeux esthétiques se déplacent et se transforment: par exemple dans le rapport entre plasticité et dispositif logique, ou avec le questionnement commun du corps et du

\footnotetext{
${ }^{7}$ Réponse de Beckett à des questions posées par un groupe de collégiens à la fin de l'opéra de La Dernière Bande, présenté à Bielefeld en février 1961. Compterendu publié dans Spectaculum, vol. 6. Francfort-sur-le-Main: Suhrkamp,1963: p.319, cité par KNOWLSON, James. Beckett. Paris: Acte sud, 1999: 607.

${ }^{8}$ Dans Qu'est-ce que la philosophie (Minuit,1991), sur l'affect et le percept, Deleuze cite Virginia Woolf et sa volonté de "saturer l'atome", à la fois pour "éliminer l'inertie" (le superflu, les déchets, les clichés) et "tout y mettre", "inclure l'absurde, les faits, le sordide mais traités par transparence”. WOOLF, Virginia. Journal d'un écrivain. Tome I, Paris: 10-18, 1974: 230.
} 
statut même de l'œuvre ouvert à d'autres supports, à d'autres techniques - autant d'enjeux développés de manière particulièrement riche dans certaines œuvres contemporaines. C'est ainsi que l'écriture met l'interprétation à l'épreuve d'une "transversalité"* (Deleuze sur Proust). Non seulement d'un symptôme à l'autre donc, mais aussi d'une œuvre de Beckett à l'autre: Imagination morte imaginez est la "matrice" du Dépeupleur ou même d'un texte comme Compagnie (1985) - si l'on remplace l'éblouissement intermittent par les bribes de "souvenirs". Passage hors de l'œuvre de Beckett enfin, et d'un domaine formel à l'autre: de Dante à Dan Graham, du travail d'écriture poétique à la vidéo, la peinture, les installations ou le théâtre contemporain. C'est à cette mobilité permanente que nous oblige l'œuvre de Beckett, écriture plurielle qui sans cesse circule et se risque à faire signe depuis l'inassignable.

\section{Guillaume Gesvret (Paris 7)}

Doutorando em Letras Modernas na Universidade de Paris 7 Denis Diderot, prepara uma tese sobre as questôes de plasticidade na obra de Beckett e as artes contemporâneas, sob a orientação de Evelyne Grossman.

\section{Resumo}

Em uma extrema concentração de meios, o curto texto Imagination morte imaginez (1966) de Samuel Beckett propõe uma experimentação a um só tempo poética e plástica. A definição estável dos corpos e das identidades, e até mesmo do próprio estatuto da obra, acha-se aí posta em crise, sendo incessantemente reinventada. Entre afeto e plasticidade, um excesso se afirma permitindo compreender de outro modo o minimalismo de Beckett e a saturação paradoxal cuja leitura o escritor nos propõe.
*(DELEUZE, Gilles. Proust et les signes. Paris: PUF, 1964: 202.)
Palavras-chave: Samuel Beckett; excesso; afeto; plasticidade. 
Key words: Samuel Beckett;
excess; affect; plasticity.

Mots-clés: Samuel Beckett; excès; affect; plasticité.
Abstract

The short text Imagination Dead Imagine (1966) by Samuel Beckett offers a both poetic and plastic experiment. The clear definition of bodies and identities, or even of the status of the work, is questioned and constantly reinvented. Between affect and plasticity, an excess appears which leads us to understand Beckett's minimalism otherwise, and to achieve, through its reading, a paradoxical saturation.

\section{Résumé}

Dans une extrême concentration de moyens, le court texte Imagination morte imaginez (1966) de Samuel Beckett propose une expérimentation à la fois poétique et plastique. La définition stable des corps et des identités, voire du statut même de l'œuvre, y est mise en crise en ne cessant d'être réinventée. Entre affect et plasticité, un excès s'y affirme qui permet de comprendre autrement le minimalisme de Beckett et la saturation paradoxale qu'il nous donne à lire. 